Ioná Carreno ${ }^{1}$

Claudete Moreschi ${ }^{2}$

Bruna Marina ${ }^{1}$

Deise Juliana Beckel Hendges ${ }^{2}$

Claudete Rempel ${ }^{2}$

Monica Maria Celestina de Oliveira ${ }^{3}$

${ }^{1}$ Centro de Ciências Biológicas e da Saúde, Centro Universitário Univates.

R. Avelino Tallini 171, Universitário. 95900000 Lajeado RS Brasil. icarreno@univates.br ${ }^{2}$ Programa de Pós-

Graduação em Ambiente e Desenvolvimento, Centro Universitário Univates.

${ }^{3}$ Departamento de Saúde Coletiva, Universidade Federal de Ciências da Saúde de Porto Alegre.

\title{
Análise da utilização das informações do Sistema de Informação de Atenção Básica (SIAB): uma revisão integrativa
}

\author{
Analysis of the use of data from the Primary Health Care \\ Information System (SIAB): an integrative review of the literature
}

Abstract This paper aimed to identify instances of the use of data from the Primary Health Care Information System in the existing scientific literature. This study is an Integrative Literature Review and the search for articles was performed in May, 2013 in the Virtual Healthcare Library (MedLine, Lilacs and SciELO), considering publications from 2008 to 2012. The search for articles initially located 164 texts, though after applying the inclusion and exclusion criteria, this review concentrated on 12 articles. The main results showed that there are fragilities like the use of the form just for the record and the insufficient training of the teams. However, the positive aspect is that the Primary Health Care Information System is the leading source of health information and statistics, and that its information can and must be used for health planning activities. Thus, it is an information system that assists municipal management and the actions in health of its teams, alerting the community to the need for care of individual health.

Key words Primary health care indicators, Public health, Primary health care, Public health policies
Resumo O presente estudo objetivou identificar na produção científica existente, as ações de utilização das informações do Sistema de Informação de Atenção Básica. Trata-se de uma Revisão Integrativa de literatura, a busca pelos artigos foi realizada em maio de 2013 na Biblioteca Virtual de Saúde (MedLine, Lilacs e Scielo), considerando publicações de 2008 a 2012. A busca pelas produções resultou inicialmente 164 produções e a partir do estabelecimento dos critérios de inclusão e exclusão, o corpus desta revisão constituiu-se em 12 artigos. Os principais resultados evidenciaram que existem fragilidades como o uso do formulário apenas para registro e capacitação insuficiente das equipes; como potencialidades foi referido o SIAB como principal sistema de informação da saúde, e que suas informações podem e devem ser utilizados para planejamento das ações de saúde. Desta forma, o SIAB é um sistema de informação que auxilia a gestão municipal e as ações em saúde das equipes, qualificando a atenção à saúde da comunidade.

Palavras-chave Indicadores básicos de saúde, Saúde coletiva, Atenção Primária à Saúde, Políticas públicas de saúde 


\section{Introdução}

Frente à expansão da Estratégia da Saúde da Família (ESF) e discussão das questões relacionadas ao montante de dados coletados por estas equipes, teve-se a necessidade da criação de um sistema de informação que conseguisse contemplar a complexidade da organização da Atenção Primária à Saúde ${ }^{1}$.

Por esse motivo, no ano de 1998 foi implantado o Sistema de Informação da Atenção Básica (SIAB) pelo Departamento da Atenção Básica/ Secretaria da Atenção à Saúde, em parceria com o Departamento de Informática e Informação do Sistema Único de Saúde (DATASUS). As informações que compõe o banco de dados do SIAB são coletadas nos domicílios em áreas cobertas pelo Programa de Agentes Comunitários de Saúde (PACS) e ESF. Tais informações são recolhidas por meio de fichas de cadastramento e de acompanhamento que devem ser analisadas com base em relatórios específicos ${ }^{2}$.

O SIAB foi desenvolvido com o propósito de dar suporte operacional e gerencial ao trabalho de coleta de dados da ESF, gerar informações para os gestores, auxiliar e agilizar o processo de tomada de decisões, baseando-se nas necessidades desta população ${ }^{3}$. Este sistema de informação é diferente de outros sistemas caracterizando-se por ser um sistema territorializado. Por meio do SIAB, é possível descrever a realidade socioeconômica, sinalizar a situação de adoecimento e morte na população, avaliar a adequação dos serviços e ações de saúde, além de contribuir para o monitoramento da situação de saúde em áreas geográficas definidas ${ }^{2}$.

Este sistema facilita a realização do diagnóstico de saúde, norteando o planejamento e avaliação de ações em saúde das equipes ${ }^{4}$. Destaca-se a importância do SIAB para subsidiar melhores e mais precisas decisões na área da saúde, possibilitando o envolvimento dos gestores, trabalhadores e comunidade ${ }^{5}$.

Frente ao exposto e reconhecendo a importância dos dados coletados pelo SIAB, bem como a necessidade de atualização da produção científica, a fim de aprofundar conhecimentos sobre a temática e o fortalecimento do sistema, buscouse realizar uma Revisão Integrativa de literatura com o objetivo de identificar na produção científica existente as ações de utilização das informações do SIAB.

\section{Metodologia}

Trata-se de um estudo de Revisão Integrativa $(\mathrm{RI})^{6}$, no qual a busca pelos artigos foi realizada utilizando-se as palavras "SIAB" OR "Sistemas de Informação de Atenção Básica”, na Biblioteca Virtual de Saúde (BVS-Bireme), pelas bases de dados: Medical Literature Analysis and Retrieval System Online (MedLine), Literatura Latino -Americana e do Caribe em Ciências da Saúde (Lilacs) e portal da Scientific Eletronic Library Online (Scielo).

Essa busca procedeu-se em maio de 2013, a partir da leitura dos artigos, norteada pela seguinte questão: Como estão sendo desenvolvidas as ações de utilização das informações do Sistema de Informação de Atenção Básica?

O período de publicação dos artigos foi de 2008 a 2012, devido à necessidade de se avaliar como estão sendo realizadas as produções científicas acerca do SIAB após 10 anos de sua implantação, considerando que a implantação de um sistema requer um tempo de adaptação. A busca inicial foi composta por 164 produções.

Os critérios de inclusão foram: artigos que abordam a temática pesquisada, com disponibilidade online e gratuitamente do texto na integra, nos idiomas português, inglês e espanhol, publicado em periódicos nacional e internacional com classificação de Qualis A1 a B4. Foram excluídos os estudos que utilizaram o SIAB somente como fonte de dados para determinar a prevalência da condição referida de uma determinada população. Os artigos duplicados foram contados somente uma vez.

Para o acesso ao texto completo, foram usados os seguintes recursos: link disponível diretamente na própria base de dados selecionada, busca no portal do periódico em que o artigo foi publicado, busca no portal Capes.

A busca pelas produções resultou inicialmente nos seguintes números nas referidas bases de dados: 48 artigos/produções científicas na MedLine, 93 na Lilacs e 23 na Scielo, totalizando 164 produções. Por fim, a partir do estabelecimento dos critérios de inclusão e exclusão, o corpus desta revisão constituiu-se em 5 artigos na Lilacs e 7 na Scielo, totalizando 12 artigos.

A primeira etapa de análise do material foi realizada por meio de leitura e construção do quadro sinóptico. Para construção do quadro foram extraídas as seguintes variáveis: número, base de dados ou portal, autor(s), título, periódico, ano, procedência dos estudos e delineamento da pesquisa. Após, desenvolveu-se a análise de con- 
teúdo por categorização proposto por Bardin ${ }^{7}$. Posteriormente, na fase de interpretação dos resultados, foram observadas as convergências e divergências existentes à luz de diferentes autores.

Quanto aos aspectos éticos, ressalta-se que os preceitos de autoria e as citações dos autores das publicações que constituíram a amostra foram respeitados.

\section{Resultados e discussão}

$\mathrm{Na}$ presente revisão integrativa, analisaram-se 12 artigos que contemplaram a questão norteadora e os critérios de inclusão, sendo que eles foram analisados na íntegra, a fim de caracterizá-los, interpretá-los e discuti-los. Inicialmente serão apresentados e caracterizados os artigos selecionados por meio do Quadro 1. Após, serão apresentados os principais resultados encontrados nos artigos selecionados através de categorias temáticas que nortearam a produção de conhecimento sobre o SIAB.

Entre os doze (12) artigos selecionados e analisados, encontrou-se uma (1) publicação na Revista Brasileira de Saúde Materno Infantil; um (1) artigo na Revista Brasileira de Enfermagem; duas (2) produções na Revista Gaúcha de Enfermagem; um (1) estudo na Revista Ciência \& Saúde; um (1) artigo na Coletiva Acta Paulista de Enfermagem; uma (1) produção na Physis: Revista de Saúde Coletiva; dois (2) artigos na Revista de Aps - Atenção Primária à Saúde; duas (2) publicações no Caderno de Saúde Coletiva; e, um (1) artigo na Revista Online Brazilian Journal of Nursing. Quanto ao ano das publicações, verificou-se que não há artigos do ano de 2008 que se adequam aos critérios de inclusão, no entanto há um (1) do ano de 2009, cinco (5) de 2010, dois (2) de 2011 e quatro (4) produções do ano de 2012. O número de autores por artigo variou entre dois (2) e seis (6), não havendo repetição de autores nas publicações. Em relação ao delineamento de pesquisa, dois (2) artigos caracterizaram como sendo estudo quantitativo, oito (8) como estudos qualitativos e dois (2) deles foram considerados qualiquantitativos. No que diz respeito à procedência dos estudos, identificou-se que houveram três (3) estudos nos estados do Rio Grande do Sul e São Paulo, e apenas um (1) nos estados do Espírito Santo, Mato Grosso, Minas Gerais, Paraíba, Paraná e Pernambuco. Dos estudos analisados nenhum foi realizado na região Norte do país, apenas um (1) foi realizado na região Centro-Oeste, dois (2) na região Nor- deste, cinco (5) na região Sudeste e quatro (4) na região Sul (Quadro 1).

Ao evidenciar as ações de utilização das informações do SIAB, foi possível identificar as seguintes categorias: fragilidades; potencialidades e estratégias.

\section{Fragilidades na utilização \\ das informações do SIAB}

Analisando os artigos desta revisão foram constatadas diversas dificuldades e fragilidades no processo de produção da informação. No artigo II foram evidenciadas dificuldades dos ACS no preenchimento, nomeação e compreensão do número de variáveis/termos/patologias descritas nas fichas. Para que isso não ocorra, os profissionais devem ser capacitados para conhecer o programa em sua totalidade, para que dessa forma se possa fazer o preenchimento adequado das fichas, para assim buscar informações sobre a comunidade, realizar a produção e a análise de relatórios ${ }^{5}$.

Conforme os artigos IX e X, o SIAB não alcançou adequadamente o objetivo de ser um instrumento de reorganização das práticas de trabalho, sendo que sua utilização parece limitada ao registro. O SIAB é um facilitador dos processos de trabalho na ESF, no entanto, para ser usado em sua plenitude, é imprescindível que ocorram mudanças em relação à formação dos trabalhadores e ao apoio oferecido pelo estado e demais esferas 5 .

$\mathrm{O}$ artigo XI, também ratifica esta ideia de que a utilização do SIAB resume-se ao preenchimento de fichas e elaboração de relatórios. As informações não têm subsidiado planejamento e avaliação das ações, que tampouco foram incorporadas na rotina dos serviços. Segundo a percepção de alguns membros de equipes de saúde, identificou-se que o SIAB não alcançou adequadamente o objetivo de ser um instrumento de reorganização das práticas de trabalho. Ele é o principal sistema informatizado de que dispõem, mas basicamente só é alimentado ${ }^{8}$.

O artigo III cita que os profissionais de saúde não têm utilizado o SIAB para o planejamento e a avaliação das ações desenvolvidas. Esta comprovação se obteve após investigar o uso do SIAB por profissionais de Equipe de Saúde da Família, afirmando que a falta de supervisão sistematizada do preenchimento das fichas do SIAB pelos ACS, somados à falta de capacitação dos profissionais, têm contribuído para a baixa confiabilidade dos dados contidos no SIAB. 
Quadro 1. Fontes bibliográficas incluídas na revisão integrativa, segundo base de dados consultadas, autor(es), título, periódico, ano, procedência dos estudos e delineamento da pesquisa.

\begin{tabular}{|c|c|c|c|c|c|c|c|}
\hline No & $\begin{array}{c}\text { Base de dados } \\
\text { ou portal }\end{array}$ & Autor(s) & Título do trabalho & Periódico & Ano & $\begin{array}{l}\text { Procedência } \\
\text { dos estudos }\end{array}$ & $\begin{array}{l}\text { Delineamento } \\
\text { da pesquisa }\end{array}$ \\
\hline I & SCIELO & $\begin{array}{l}\text { Frias, P.G.; } \\
\text { Cavalcanti, } \\
\text { M.R.B.A.; } \\
\text { Mullachery, P. } \\
\text { H.; Nogueira, } \\
\text { G.; Damacena, } \\
\text { G. N.; } \\
\text { Szwarcwald, } \\
\text { C. L. }\end{array}$ & $\begin{array}{l}\text { Avaliação da notificação } \\
\text { de óbitos de menores } \\
\text { de um ano ao Sistema } \\
\text { de Informações da } \\
\text { Atenção Básica (SIAB). }\end{array}$ & $\begin{array}{l}\text { Revista } \\
\text { Brasileira } \\
\text { de Saúde } \\
\text { Materno } \\
\text { Infantil }\end{array}$ & 2012 & Pernambuco & Qualiquantitativa \\
\hline II & SCIELO & $\begin{array}{l}\text { Lima, A. P.; } \\
\text { Correa, A. C. } \\
\text { P.; Oliveira, } \\
\text { Q.C. }\end{array}$ & $\begin{array}{l}\text { Conhecimento de } \\
\text { Agentes Comunitários } \\
\text { de Saúde sobre os } \\
\text { instrumentos de coleta } \\
\text { de dados do SIAB. }\end{array}$ & $\begin{array}{l}\text { Revista } \\
\text { Brasileira de } \\
\text { Enfermagem }\end{array}$ & 2012 & Mato Grosso & Qualitativa \\
\hline III & SCIELO & $\begin{array}{c}\text { Marcolino, J. } \\
\text { S.; Scochi, M. J. }\end{array}$ & $\begin{array}{l}\text { Informações em } \\
\text { saúde: o uso do SIAB } \\
\text { pelos profissionais das } \\
\text { Equipes de Saúde da } \\
\text { Família. }\end{array}$ & $\begin{array}{l}\text { Revista } \\
\text { Gaúcha de } \\
\text { Enfermagem }\end{array}$ & 2010 & Paraná & Qualitativa \\
\hline IV & SCIELO & $\begin{array}{l}\text { Duarte, } \\
\text { M. L.C.; } \\
\text { Tedesco, J. R.; } \\
\text { Parcianello, } \\
\text { R. R. }\end{array}$ & $\begin{array}{l}\text { O uso do sistema } \\
\text { de informação na } \\
\text { estratégia saúde da } \\
\text { família: percepções dos } \\
\text { enfermeiros. }\end{array}$ & $\begin{array}{l}\text { Revista } \\
\text { Gaúcha de } \\
\text { Enfermagem }\end{array}$ & 2012 & $\begin{array}{l}\text { Rio Grande } \\
\text { do Sul }\end{array}$ & Qualitativa \\
\hline $\mathrm{V}$ & SCIELO & $\begin{array}{l}\text { Ferreira, M. } \\
\text { E.V.; Schimith, } \\
\text { M.D.; Caceres, } \\
\text { N. C. }\end{array}$ & $\begin{array}{l}\text { Necessidades de } \\
\text { capacitação e } \\
\text { aperfeiçoamento dos } \\
\text { profissionais de equipes } \\
\text { de saúde da família } \\
\text { da } 4^{\text {a }} \text { Coordenadoria } \\
\text { Regional de Saúde do } \\
\text { Estado do Rio Grande } \\
\text { do Sul. }\end{array}$ & $\begin{array}{l}\text { Ciência e } \\
\text { Saúde Coletiva }\end{array}$ & 2010 & $\begin{array}{l}\text { Rio Grande } \\
\text { do Sul }\end{array}$ & Qualiquantitativa \\
\hline VI & SCIELO & $\begin{array}{l}\text { Souza, J.; Luis, } \\
\text { M. A. V. }\end{array}$ & $\begin{array}{l}\text { Demandas de saúde } \\
\text { mental: percepção de } \\
\text { enfermeiros de equipes } \\
\text { de saúde da família. }\end{array}$ & $\begin{array}{l}\text { Acta } \\
\text { Paulista de } \\
\text { Enfermagem }\end{array}$ & 2012 & São Paulo & Qualitativa \\
\hline
\end{tabular}

continu

Como exemplo dessa situação, o artigo I apresenta uma notificação excessiva de óbitos em crianças menores de um ano de idade cadastradas no SIAB. Destes óbitos registrados, somente $34 \%$ foram confirmados pelos ACS que são os profissionais responsáveis pelas coletas de dados e preenchimento das fichas do SIAB. Apesar disso, ressalta-se a importância do SIAB como instrumento de reorganização do processo de trabalho, mesmo que sua utilização pela ESF tenha se mostrado simples, quando comparada às suas possibilidades de uso ${ }^{8}$. 
Quadro 1. continuação

\begin{tabular}{|c|c|c|c|c|c|c|c|}
\hline No & $\begin{array}{c}\text { Base de dados } \\
\text { ou portal }\end{array}$ & Autor(s) & Título do trabalho & Periódico & Ano & $\begin{array}{l}\text { Procedência } \\
\text { dos estudos }\end{array}$ & $\begin{array}{c}\text { Delineamento } \\
\text { da pesquisa }\end{array}$ \\
\hline VII & SCIELO & $\begin{array}{l}\text { Addum, F. M.; } \\
\text { Serra, C.G .; } \\
\text { Sessa, K.S.; } \\
\text { Izoton, L.M.; } \\
\text { Santos, T.B. }\end{array}$ & $\begin{array}{l}\text { Planejamento local, } \\
\text { Saúde Ambiental e } \\
\text { Estratégia Saúde da } \\
\text { Família: uma análise } \\
\text { do uso de ferramentas } \\
\text { de gestão para a } \\
\text { redução do risco de } \\
\text { contaminação por } \\
\text { enteroparasitoses no } \\
\text { município de Venda } \\
\text { Nova do Imigrante. }\end{array}$ & $\begin{array}{l}\text { Physis: Revista } \\
\text { de Saúde } \\
\text { Coletiva }\end{array}$ & 2011 & Espírito Santo & Qualitativa \\
\hline VIII & LILACS & $\begin{array}{c}\text { Roese, A.; } \\
\text { Pinto, J.M.; } \\
\text { Gerhardt, T. E.; } \\
\text { Silveira, D. T. }\end{array}$ & $\begin{array}{l}\text { Perfil de hipertensão } \\
\text { arterial sistêmica e } \\
\text { de diabetes mellitus } \\
\text { a partir de bases de } \\
\text { dados nacionais em } \\
\text { municípios de pequeno } \\
\text { porte no Rio Grande do } \\
\text { Sul, Brasil. }\end{array}$ & $\begin{array}{l}\text { Revista de } \\
\text { APS - Atenção } \\
\text { Primária à } \\
\text { Saúde }\end{array}$ & 2011 & $\begin{array}{l}\text { Rio Grande } \\
\text { do Sul }\end{array}$ & Quantitativa \\
\hline IX & LILACS & $\begin{array}{l}\text { Barbosa, D. C. } \\
\text { M.; Forster, } \\
\text { A. C. }\end{array}$ & $\begin{array}{l}\text { Sistemas de Informação } \\
\text { em Saúde: a perspectiva } \\
\text { e a avaliação dos } \\
\text { profissionais envolvidos } \\
\text { na Atenção Primária } \\
\text { à Saúde de Ribeirão } \\
\text { Preto, São Paulo. }\end{array}$ & $\begin{array}{l}\text { Caderno de } \\
\text { Saúde Coletiva }\end{array}$ & 2010 & São Paulo & Qualitativa \\
\hline $\mathrm{X}$ & LILACS & $\begin{array}{l}\text { Figueiredo, L. } \\
\text { A.; Pinto, I. C.; } \\
\text { Marciliano, } \\
\text { C. S. M.; } \\
\text { Souza, M. de } \\
\text { F.; Guedes, A. } \\
\text { A. B }\end{array}$ & $\begin{array}{l}\text { Análise da utilização } \\
\text { do SIAB por quatro } \\
\text { equipes da estratégia } \\
\text { saúde da família do } \\
\text { município de Ribeirão } \\
\text { Preto, SP. }\end{array}$ & $\begin{array}{l}\text { Caderno de } \\
\text { Saúde Coletiva }\end{array}$ & 2010 & São Paulo & Qualitativa \\
\hline $\mathrm{XI}$ & LILACS & $\begin{array}{l}\text { Lima, R. T. de; } \\
\text { Costa, G. M. } \\
\text { C.; FRANÇA, } \\
\text { I. S. X. de; } \\
\text { SOUSA, F. S. } \\
\text { de; COURA, } \\
\text { A. S. }\end{array}$ & $\begin{array}{l}\text { O sistema de } \\
\text { informação como } \\
\text { ferramenta para } \\
\text { planejamento e } \\
\text { avaliação dos serviços } \\
\text { de saúde: estudo } \\
\text { descritivo. }\end{array}$ & Revista OBJN & 2010 & $\begin{array}{l}\text { Paraíba } \\
\text { (Campina } \\
\text { Grande) }\end{array}$ & Qualitativa \\
\hline XII & LILACS & $\begin{array}{l}\text { Tibiriçá, S. H. } \\
\text { C.; Ezequiel, } \\
\text { O. da S.; } \\
\text { Carminate, D. } \\
\text { la G.; Rioga, } \\
\text { G. das M. P.; } \\
\text { Ribeiro, L. C.; } \\
\text { Teixeira, M. } \\
\text { T. B. }\end{array}$ & $\begin{array}{l}\text { O potencial do cadastro } \\
\text { das famílias no Sistema } \\
\text { de Informação da } \\
\text { Atenção Básica em } \\
\text { saúde, como base } \\
\text { para determinação da } \\
\text { amostra em pesquisas } \\
\text { na área da saúde. }\end{array}$ & $\begin{array}{l}\text { Revista de } \\
\text { APS - Atenção } \\
\text { Primária à } \\
\text { Saúde }\end{array}$ & 2009 & Minas Gerais & Quantitativa \\
\hline
\end{tabular}


$\mathrm{O}$ artigo VIII constatou que os profissionais coletam e preenchem um grande número de dados, os quais são encaminhados às Secretarias Municipais de Saúde. Porém, estes não recebem retorno das informações enviadas, fato que acaba desmotivando os profissionais em realizar corretamente o cadastro do SIAB. Os ACS são os profissionais que mais usam o SIAB, porém, a falta de discussão e utilização das informações pelo restante da equipe faz com que as informações coletadas sejam pouco valorizadas ${ }^{4}$.

Conforme o artigo IV, a falta de apoio/assessoria para tirar dúvidas, bem como a falta de abordagem deste conhecimento durante a graduação dos profissionais de saúde, representam limitações para o manuseio do sistema. Cada profissional realiza as anotações dos dados de uma forma, para suprir suas próprias dificuldades. Uma questão a ser repensada é a forma como as instituições formadoras estão capacitando estes profissionais para o trabalho em equipe ${ }^{4}$.

No artigo XI foi constatada a falta de supervisão, controle e análise da qualidade dos dados produzidos por parte de instâncias superiores. $\mathrm{E}$, dentre as dificuldades encontradas no manuseio do SIAB, estão àquelas relacionadas à falta de flexibilidade, ao número limitado de doenças ou condições referidas na ficha e à ausência de treinamentos. Outros estudos salientam a importância das Coordenadorias Regionais de Saúde na organização de capacitações e discussões sobre o SIAB, além de assessorar os municípios na implantação efetiva desse sistema. Envolvendo gestores, trabalhadores e comunidade na consolidação do SIAB facilitaria/qualificaria as ações de planejamento em saúde nas comunidades ${ }^{5}$.

$\mathrm{O}$ artigo $\mathrm{X}$ evidencia que os profissionais compreendem a finalidade do SIAB de produzir informações para auxiliar a equipe no diagnóstico da realidade local, no entanto, sua utilização se encontra limitada ao registro, não sendo o SIAB utilizado enquanto instrumento gerencial básico para o planejamento e a avaliação das ações de promoção, prevenção e reabilitação em saúde e da transformação da situação local.

Para promover a efetividade do SIAB, a educação permanente, bem como a avaliação constante das dificuldades de sua utilização como um instrumento para a programação do trabalho da equipe de saúde da família são requisitos fundamentais para se alcançar os objetivos e as metas propostas pelo sistema. Somente o acesso aos manuais é insuficiente, precisa-se investir em capacitação. Para isso é necessário o envolvimento das três esferas administrativas do SUS, de modo a efetivar e consolidar parcerias com o objetivo de promover a educação permanente, realizando oficinas, fóruns, grupos de estudos, palestras e atividades intersetoriais 9 .

Os artigos VI e VII apresentam a carência de novos investigadores. No artigo VI, profissionais de equipes de ESF apontaram a necessidade do SIAB incluir um indicador específico para as demandas de saúde mental, tendo em vista que este contribuirá para sua sistematização em nível territorial. Já que o SIAB tem os indicadores de diabetes, hipertensão, gestantes e crianças, os quais facilitam o levantamento e a localização das famílias com tais necessidades, seria viável a inclusão da saúde mental no processo de investigação.

Segundo o artigo VII, isto também ocorre no que se refere às características ambientais, na qual a Ficha A do SIAB limita o meio ambiente em coleta de esgoto, coleta de água, recolhimento de lixo, tipo de casa e energia. Tal limitação não permite uma avaliação completa da residência, comprometendo a eficácia do planejamento local em relação à saúde ambiental. O estudo observou que o SIAB deveria ser informatizado e que deve haver uma atualização nas fichas, além disso, a equipe deveria ser treinada e capacitada para atuar de maneira adequada ${ }^{9}$.

A presença incipiente de uma educação permanente mostra a falta de uma política de qualificação dos profissionais nos municípios estudados, transparecendo as fragilidades do sistema e trazendo consequências para a qualidade das informações coletadas e analisadas ${ }^{5}$.

\section{Potencialidades da utilização das informações do SIAB}

$\mathrm{Na}$ análise dos artigos observaram-se potencialidades de utilização das informações contidas no SIAB. O artigo I refere que o SIAB permite a disponibilidade de indicadores, monitoramento e avaliação da atenção primária, o que corrobora com os artigos IV e IX, os quais citam que esse sistema serve como instrumento para coleta de dados, controle e informações que auxiliem no diagnóstico local. Sendo considerado o principal sistema de informação da saúde, se diferenciando dos demais por possibilitar a utilização pontual das informações, produção de indicadores e consolidação progressiva da informação.

O sistema de informação mostra, por meio de números, a realidade de saúde de um determinado município ${ }^{10}$. O SIAB é uma ferramenta de trabalho essencial na ESF e serve para sistematizar os dados levantados da comunidade da área 
adscrita, otimizando o trabalho da equipe ${ }^{11}$. Este sistema, além de representar um instrumento de trabalho das equipes da ESF, é útil na identificação das famílias e de prioridades, na organização do trabalho, na programação local e no direcionamento das visitas domiciliares ${ }^{12}$.

$\mathrm{O}$ artigo IV apontou o SIAB como um sistema de extrema importância, o qual facilita a coleta de dados da população pelos profissionais da ESF. O estudo o considera um instrumento de controle de saúde da comunidade da área de abrangência das equipes da ESF, onde os próprios profissionais usufruem desses dados coletados para o planejamento das ações de saúde. Assim, através da identificação das necessidades e das condições de saúde das famílias, existe a possibilidade de criação de vínculo entre profissionais e usuários de saúde. Esse encontro propicia um cuidado no domicílio, indo além das ações realizadas no serviço.

$\mathrm{O}$ artigo VII ressalta que a proximidade que os profissionais têm com a população é mais importante do que o preenchimento do papel, pois por meio deles é possível saber quem é cada usuário, caso haja necessidade de realizar alguma alteração.

Ainda, no artigo IV o SIAB apresenta-se como um instrumento para coleta de dados, de controle e de informações, além de ser uma ferramenta que auxilia no diagnóstico local, ajudando na identificação das necessidades de saúde da comunidade, o qual foi percebido como um facilitador dos processos de trabalho na ESF. Outro estudo mostra que um sistema de informação em saúde auxilia a formular e avaliar as políticas, planos e programas de saúde, formando uma ferramenta para tomada de decisões, objetivando a melhoria da situação da população ${ }^{12}$.

Conforme o artigo X, o SIAB é importante para a organização do serviço, pois traz dados significativos que possibilitam o direcionamento do processo de trabalho das equipes da ESF. Se o SIAB for utilizado de maneira apropriada pela gestão local e municipal, é um elemento fundamental para planejamentos, monitoramentos e avaliações das ações em saúde na ESF ${ }^{13}$.

Segundo o artigo VIII os dados do SIAB são coerentes com a realidade abrangida pela ESF. As observações documentadas no estudo XII revelaram a importância do SIAB, não somente como gerador de dados que subsidiam as políticas do Programa Saúde da Família (PSF), mas como ferramenta eficaz para disponibilizar cadastros fidedignos a serem utilizados, tanto em pesquisas na APS, como nas diversas áreas das ciências da saúde. O SIAB consolida os dados da ESF, onde para a análise dos dados referentes à população cadastrada deve-se considerar que os indicadores informados são proporcionais à sua cobertura ${ }^{10}$.

$\mathrm{O}$ artigo XII avaliou a cobertura do cadastro das famílias através da comparação entre a ficha A aplicada pelo ACS e os dados obtidos de investigações realizadas diretamente nos domicílios, validando a confiabilidade do cadastro realizado pelo PSF, justificando sua utilização como ferramenta legítima para determinação de amostras nas pesquisas básicas e clínicas.

\section{Estratégias para utilização das informações do SIAB}

Neste estudo é imprescindível destacar as estratégias apresentadas nos artigos analisados, visando uma melhor utilização das informações do SIAB. Os artigos I, II e VII citam que devem ser ofertados cursos introdutórios aos profissionais de saúde para promover o aprimoramento do preenchimento das fichas. O artigo IX traz que os problemas operacionais e as falhas de uso do software poderiam ser otimizadas ou resolvidas por meio de uma equipe treinada adequadamente. Ainda, o artigo I pontua a importância de promover momentos de análise e discussão dos relatórios emitidos pelo sistema.

Além disso, os estudos II, III e X mencionam a importância de formular e promover ações de educação permanente em saúde aos profissionais que atuam na ESF. É necessário que haja investimento por parte dos gestores em ações educativas voltadas ao aperfeiçoamento do manuseio do cadastro pelos profissionais envolvidos. A educação permanente e a avaliação contínua das dificuldades de utilização do SIAB são requisitos fundamentais para se alcançar os objetivos e as metas propostas pela ESF ${ }^{9}$. Segundo o artigo IV, os profissionais necessitam conhecer o sistema de informação e saber utilizá-lo, para isso é necessário que aconteçam mudanças em relação à formação dos trabalhadores e ao apoio oferecido pelo estado e demais esferas

Segundo o estudo IV os trabalhadores devem ser capacitados para conhecer o programa, no qual a educação permanente e a promoção de uma política para qualificação dos profissionais são essenciais na obtenção da qualidade das informações coletadas e analisadas. Ressalta-se em estudo que a falta de capacitação devido à ausência de treinamentos para o adequado preenchimento das fichas e uso de material impresso é referida como sendo prejudicial à confiabilida- 
de dos dados e à qualidade da informação ${ }^{14}$. Os autores do artigo IV ainda citam que há necessidade de assessoria das Coordenadorias Regionais de Saúde na organização de capacitações e discussões sobre o SIAB, além de assessorar os municípios na implantação efetiva desse sistema na atenção básica, envolvendo gestores, trabalhadores e comunidade.

$\mathrm{O}$ artigo III cita que na formação dos profissionais, tanto de nível médio como superior, deveriam ser utilizadas estratégias de ensino que possibilitem o trabalho em equipe multiprofissional, a valorização da informação para o planejamento das ações e a melhoria da supervisão do processo de trabalho. As ações mais indicadas são a implementação de educação permanente, a promoção da conscientização da importância do trabalho realizado e a realização de mudanças no sistema de acordo com a realidade local para abranger as especificidades de cada região $\mathrm{O}^{14}$.

Apesar do SIAB apresentar potencialidades suficientes para servir como um instrumento de monitoramento e avaliação da atenção primária, mediante as novas necessidades de saúde, continua necessitando de ajustes quanto à sua concepção, sobretudo, quanto ao seu modo de operar ${ }^{15}$. Pois tomadas de decisões informadas por melhores evidências alcançam melhores resultados em saúde. Estratégias adequadas podem favorecer o potencial inovador e criativo nos processos de gestão, assim como as iniciativas de consolidação de práticas de formulação e implementação de políticas informadas por evidências no Brasil e no mundo ${ }^{16}$.

Para tanto, ao considerar a importância da compreensão dos indicadores e do preenchimento das fichas desse sistema, a capacitação permanente representa uma ferramenta imprescindível $^{17}$. Na perspectiva da educação permanente, para os gestores e profissionais de saúde, o aperfeiçoamento dos registros ampliados de saúde possibilita analisar de forma integrada os dados gerados pelos diferentes sistemas de informação conforme a territorialização adotada pelos municípios. Assim, considera-se premente o incentivo e a qualificação dos profissionais de saúde para a utilização destes registros ${ }^{18}$.

\section{Conclusões}

Ao identificar na produção científica existente as ações de utilização das informações do SIAB, evidenciaram-se algumas fragilidades, dentre as principais se destaca o uso deste sistema somente como um formulário para registro. Também foi possível notar que há dificuldades por parte dos profissionais de saúde no preenchimento das fichas.

Em relação ao despreparo das equipes para a utilização do SIAB, fica evidente que tal problemática decorre da capacitação insuficiente das equipes, já que o treinamento introdutório não discute de forma aprofundada o SIAB, sendo o preparo das ESF insuficiente para o preenchimento das fichas, discussão de suas variáveis e utilização do sistema para a extração de relatórios com o objetivo de auxiliar no planejamento das ações. Quanto às limitações das fichas, os dados levantados evidenciaram que as fichas do SIAB apresentam aspectos limitantes, como no caso de dados referentes ao meio ambiente e à saúde mental da população cadastrada.

O SIAB é um sistema que permite a visualização do perfil epidemiológico e sanitário da população de sua área de abrangência, oferecendo um diagnóstico da situação de saúde e, mesmo diante das fragilidades identificadas, deve-se reconhecer o valor do sistema para as equipes. Há potencialidades relacionadas ao SIAB que precisam ser destacadas, pois este é considerado o principal sistema de informação da saúde. Além de facilitar a coleta de dados da população cadastrada na ESF, os dados podem e devem ser utilizados para planejamentos das ações de saúde.

Os estudos apresentam diversas estratégias para um melhor aproveitamento do SIAB e dos dados que podem ser extraídos dele. Sugere-se que sejam feitas capacitações e treinamentos com os profissionais de saúde.

Havendo uma educação permanente de toda a equipe e assessorias por parte das Coordenadorias Regionais de Saúde, os profissionais de saúde obterão um maior conhecimento sobre este sistema. Sabendo utilizá-lo adequadamente haverá a valorização da informação para então realizar o planejamento de ações e atender a comunidade baseado na equidade e integralidade da atenção à saúde. 


\section{Colaboradores}

I Carreno, C Moreschi, B Marina e DJB Hendges participaram da concepção e delineamento, interpretação dos dados, redação do artigo, revisão crítica e aprovação da versão a ser publicada; C Rempel participou da interpretação dos dados, redação do artigo, revisão crítica e aprovação da versão a ser publicada; MMC Oliveira participou da concepção e delineamento, revisão crítica e aprovação da versão a ser publicada.

\section{Agradecimentos}

Agradecemos ao apoio do $\mathrm{CNPq}$ pela concessão da pesquisa Análise da epidemiologia genética, da situação de saúde da população cadastrada no Sistema de Informações de Atenção Básica (SIAB) e avaliação da Atenção Primária à Saúde pela percepção dos profissionais da saúde de Lajeado (RS), Brasil.

\section{Referências}

1. Brasil. Ministério da Saúde (MS). Saúde boa e vida melhor para 50 milhões. Rev Bras Saúde Fam 2002; 5(n esp):66-69.

2. Brasil. Ministério da Saúde (MS), Secretaria de Atenção à Saúde, Departamento de Atenção Básica. Sistema de Informação da Atenção Básica - SIAB: indicadores 2003. Brasília: MS; 2004.

3. França I. Sistema de Informação da Atenção Básica: um estudo exploratório [dissertação]. Rio de Janeiro: Fundação Oswaldo Cruz; 2001.

4. Marcolino JS, Scochi MJ. Informações em saúde: o uso do SIAB pelos profissionais das Equipes de Saúde da Família. Rev Gaúcha Enferm 2010; 31(2):314-320.

5. Duarte MLC, Tedesco JR, Parcianello RR. O uso do sistema de informação na estratégia saúde da família: percepções dos enfermeiros. Rev Gaúcha Enferm 2012; 33(4):111-117.

6. Mendes KDS, Silveira RCCP, Galvão CM. Revisão integrativa: método de pesquisa para a incorporação de evidências na saúde e na enfermagem. Texto Contexto Enferm 2008; 17(4):758-764.

7. Bardin L. Análise de conteúdo. Lisboa: Edições; 2009.

8. Barbosa DCM, Forster AC. Sistemas de Informação em Saúde: a perspectiva e a avaliação dos profissionais envolvidos na Atenção Primária à Saúde de Ribeirão Preto/SP. Cad Saúde Coletiva 2010; 18(3):424-433.

9. Queiroga RM, Andrade AN, Abrantes KSM, Costa TS, Sobreira MV, Casimiro GS. Aplicabilidade do Sistema de Informação da Atenção Básica no cotidiano de Enfermeiros. Rev Rene 2011; 12(n. esp.):943-951.

10. Jesus MCP, Santos SMR, Goulart TP, Martins NA, Peres RB, Coelho VS. Atualização dos dados nos Sistemas de Informação em Saúde. Rev Enferm UERJ 2012; 20(n. esp. 2):795-801

11. Lima AP, Corrêa ACP, Oliveira QC. Conhecimento de Agentes Comunitários de Saúde sobre os instrumentos de coleta de dados do SIAB. Rev Bras Enferm 2012; 65(1):121-127.

12. Figueiredo LA, Pinto IC, Marciliano CSM, Souza MF, Guedes AAB. Análise da utilização do SIAB por quatro equipes da estratégia saúde da família do município de Ribeirão Preto, SP. Cad Saude Coletiva 2010; 18(3):418423.

13. Oliveira QC, Corrêa ACP, Lima AP, Teixeira RC, Pedrosa ICF. Sistema de Informação da Atenção Básica - Situação de Saúde de um Município de Mato Grosso. Cienc Cuid Saude 2010; 9(1):36-43.

14. Radigonda B, Conchon MF, Carvalho WO, Nunes EFPA. Sistema de Informação da Atenção Básica e sua utilização pela Equipe de Saúde da Família: Uma Revisão Integrativa Rev Espaço para a Saúde 2010; 12(1):3847.

15. Frias PG, Cavalcanti MRBAC, Mullachery PH, Damacena GN, Szwarcwald CL. Avaliação da notificação de óbitos de menores de um ano ao Sistema de Informações da Atenção Básica (SIAB). Rev Bras Saúde Matern Infant 2012; 12(1):15-25.

16. Silva SF, Souza NM, Barreto JOM. Fronteiras da autonomia da gestão local de saúde: inovação, criatividade e tomada de decisão informada por evidências. Cien Saude Colet 2014; 19(11):4427-4438. 
17. Ferreira MEV, Schimith MD, Caceres NC. Necessidade de capacitação e aperfeiçoamento de profissionais de equipes de saúde da família da $4^{\text {a }}$. Coordenadoria Regional de Saúde do Estado do Rio Grande do Sul. Cien Saude Colet 2010; 15(5):2611-2620.

18. Magalhães MC, Teixeira MTB, Ribeiro LC, Moreno AB, Caetano R, Campos SEM, Camargo Júnior KR, Coeli $\mathrm{CM}$. Indicadores relacionados ao nascimento da população adscrita a duas Unidades de Saúde da Família. Cad Saude Coletiva 2011; 19(3):299-304.

Artigo apresentado em 13/09/2013

Aprovado em 05/12/2013

Versão final apresentada em 07/12/2013 